\title{
Easy Optical Defragmentation with SDN Controlled Tunable Transmitter
}

\author{
A. Dupas(1), D. Verchere(1), Q. Pham Van(1), P. Layec(1), L. Bramerie(2), A. Carer(2), B. Haentjens(3), E. \\ Le Rouzic ${ }^{(4)}$ \\ (1) Nokia Bell-Labs France, Nozay, arnaud.dupas@nokia-bell-labs.com \\ (2) University of Rennes 1, Lannion, France \\ (3) Vectrawave, Lannion, France \\ (4) Orange Labs, Lannion, France
}

\begin{abstract}
To ease automatic optical spectrum defragmentation, we present a programmable SDN controlled 100Gbit/s transmitter prototype with a fast wavelength and bitrate switching capabilities. It can be tuned in $0.5 \mathrm{~ms}$ over all C-band and used for service hitless defragmentation application.
\end{abstract}

\section{Introduction}

Elastic Optical Networks is now widely deployed since its introduction $\mathrm{in}^{1-3}$. It can save OPEX by reducing the number of opto-electronic regenerators, take advantage of new flexi-grid ROADMs and improvements in tunable laser or DSP technologies. To efficiently benefit from multiple rates in EON and to optimize the limited spectral resources over time, optical spectrum defragmentation is required. Today, we use defragmentation during maintenance windows because lightpaths must be often switched off to be able to move the wavelength over the $C$ band. In EON, defragmentation may become challenging when using different spectrum grids. To overcome this, automation is needed. Experiments were conducted in ${ }^{4}$ with an SDN control without studying optical hardware network elements.

In this paper, we propose a solution to deploy optical spectrum defragmentation automatically, with fast hardware elements and SDN control. the process may also include lightpaths rerouting without traffic interruption. We first present the architecture and depict how to allow a fast SDNbased defragmentation. We then set up an experiment and perform hardware characterizations.

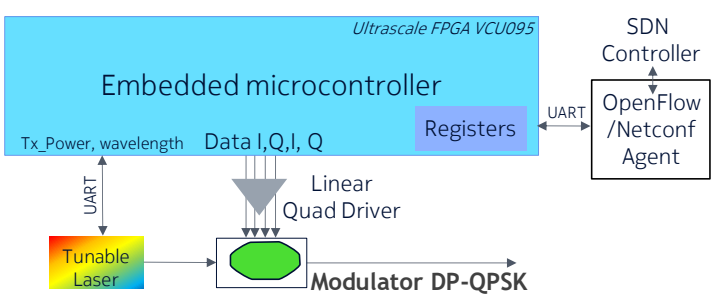

Fig. 1: Transmitter prototype architecture

\section{Software Defined Elastic Optical Network}

To perform automatic optical defragmentation in flexi-grid network, we need both hardware and software tools. We present here both. The SDN controller is built on ONOS $^{5}$ Software. ONOS contains the packages for the optical devices data models drivers. Specific extensions have been coded in the agent and in the core of the controller to take advantage of the new functionalities of this hardware, like fast bitrate switching and fast wavelength tuning. As the ONOS based network controller is modular and coded in containers (Fig. 4), it can be easily deployed and updated by an operator. Each Virtual Network Functions (VNF) executed by ONOS to control the optical nodes can be deployed locally near the hardware or in the cloud, depending on the latency and performance requirements. We present here a solution that takes advantage of both deployment and highspeed ONOS-compatible hardware. The optical defragmentation $\mathrm{VNF}^{6}$ is a composition of several optical VNF including media channel routing \& spectrum allocation. For the tests of the bandwidth variable and tuning transmitter, a network of 6 emulated optical nodes is defined and one port of one optical node is related to the elastic transmitter prototype. The reconfiguration commands are sent to the control of the nodes to configure their optical ports. The transmitter port can also be reconfigured when the defragmentation VNF changes the route and/or the spectrum assignment of the corresponding media channel.

\section{Hardware architecture of the transmitter}

The optical transmitter is composed of an elastic programmable interface and a high-speed tunable laser (Fig. 1). Both baudrate and wavelength can be adjusted. The electronic board is based on an FPGA and performs realtime aggregation of input ports connected to 10 OTU2 clients (Fig. 2). It has been proposed in $2015^{7}$ for flexi-grid restoration because the number of clients is programmable, resulting in a 
transmitted data traffic changing from $10.7 \mathrm{GBit} / \mathrm{s}$ to $107 \mathrm{Gbit} / \mathrm{s}$. A first switching time has been measured in less than $500 \mu \mathrm{s}$, and recent improvements 8 have been made to perform the data switching in about $12 \mu$ s with a repetition coding scheme. Data must be amplified through a quad linear GaAs driver to convert the I/Q frames into a coherent signal with two orthogonal polarizations via an opto-electronic MZM modulator. The quad driver is a prototype ${ }^{9}$ using SIP (System In Package) and MMIC (Microwave Monolithic Integrated Circuits) technics (Fig. 6). The 2 channels amplifier core is made on low noise $150 \mathrm{~nm}$ GaAs pHEMT process and helps to decrease the phase difference between I and $Q$ line. The TWA (Travelling Wave Amplifier) structure has been optimized to reach high linearity behavior especially for PAM4 eye shape. The continuous optical signal at the input of the MZM modulator is coming from a fast-tunable laser. It is programmed by the SDN controller through an embedded microcontroller and an Openflow / Netconf agent which role is to convert the configuration issued by the SDN controller and to send back the monitored information including the optical power and wavelength, or
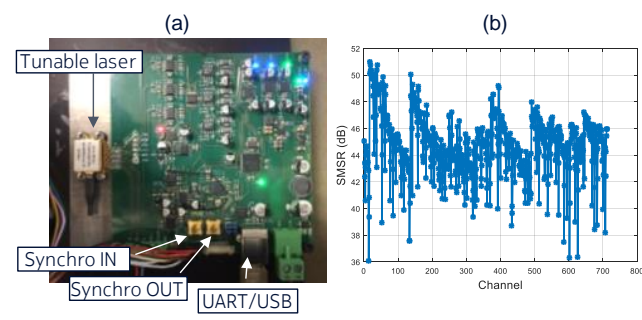

Fig. 3: Tunable laser driver prototype a specific electronic control card has been developed to control the 5 sections (insert Fig. 3.a): The SOA, Gain, phase, left and right sections. The static linewidth is maximum 10 $\mathrm{MHz}$ for all wavelength in the C-band.

To be compatible with the flexi-grid and the channel spacing of $6.25 \mathrm{Ghz}$, effort has been done on the electronic driver board to address all 705 channels. We have measured a SMSR superior to $36 \mathrm{~dB}$ for all channels (Fig. 3.b). Particular attention was paid to frequency drift related to thermal effects during wavelength switching in using preemphasis technique on phase section. The switching between any wavelength is characterized below 500 ns with

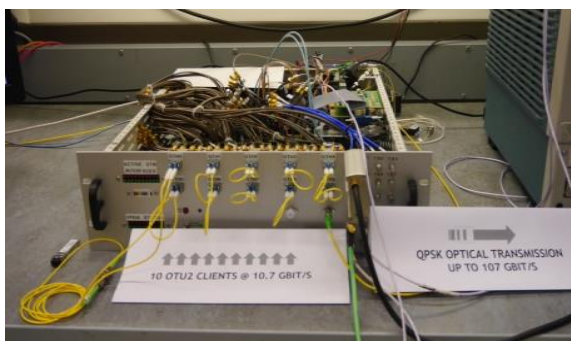

Fig. 2: Elastic transmitter prototype

frequency deviation inferior to $1 \mathrm{GHz}$. It is realized with a couple of DAC running at $100 \mathrm{MHz}$, and a high-speed switch sending DAC values to 3 laser sections. All currents values (phase, left, right sections) for all wavelengths are saved in Look-Up-Table (LUT). Only the gain and the SOA section remain constant, providing a constant, but programmable, output power.

\section{Experimental results}

status of the multiplexing process.

\section{Tunable laser driver}

The high-speed tunable laser is a Modulated

Grating $\mathrm{Y}$ laser (MG-Y). For the demonstrator,
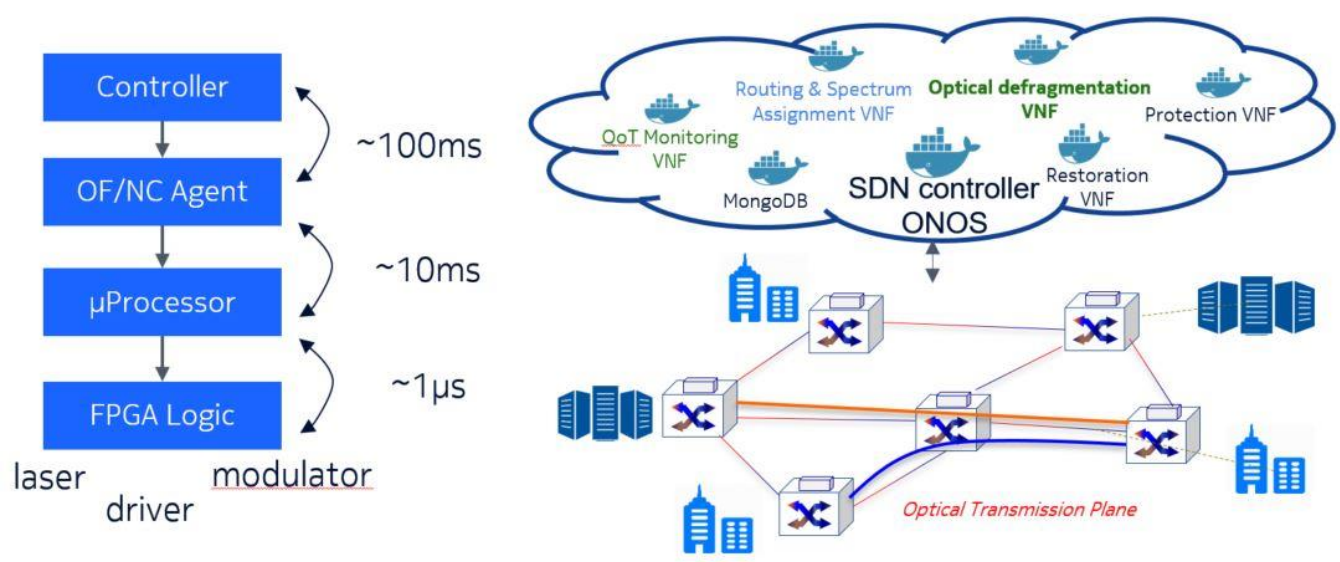

Fig. 4: SDN controller architecture with optical defragmentation VNF 
To characterize all the set-up, switching times

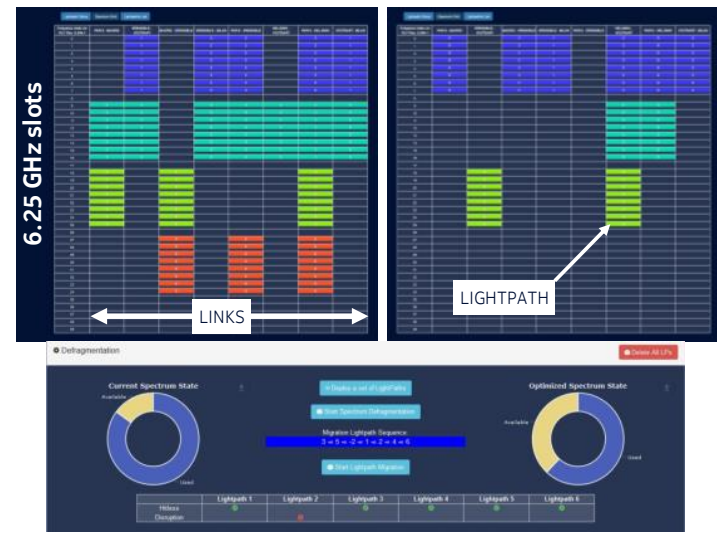

Fig. 5: Spectrum grid allocation before (above left) and after (right) execution of defragmentation VNF, GUI (below).

have been measured from the Defragmentation VNF to the effective wavelength changing (Tab. 1). $480 \mathrm{~ms}$ are required to send the command from VNF GUI (Fig 5) to the OpenFlow / Netconf agent. From the agent to the FPGA board, $12 \mathrm{~ms}$ are needed to transfer the order and decode it. Then we measured on an oscilloscope the time between the FPGA registers update and the

Tab. 1: Switching times

\begin{tabular}{|c|c|c|}
\hline $\begin{array}{c}\text { Source } \\
\text { SDN } \\
\text { Controller }\end{array}$ & Destination & Time \\
\hline Agent & El. Transmitter & $12 \mathrm{~ms}$ \\
\hline El. Transmitter & $\lambda 0$ to/from $\lambda 1$ & $403 / 365 \mathrm{~ns}$ \\
\hline El. Transmitter & $\lambda 0$ to/from $\lambda 2$ & $380 / 424 \mathrm{~ns}$ \\
\hline El. Transmitter & $\lambda 3$ to/from $\lambda 2$ & $425 / 472 \mathrm{~ns}$ \\
\hline
\end{tabular}

effective wavelength changes in the tunable laser, with a trigger output signal. Depending on the wavelength couple 'begin/end', all
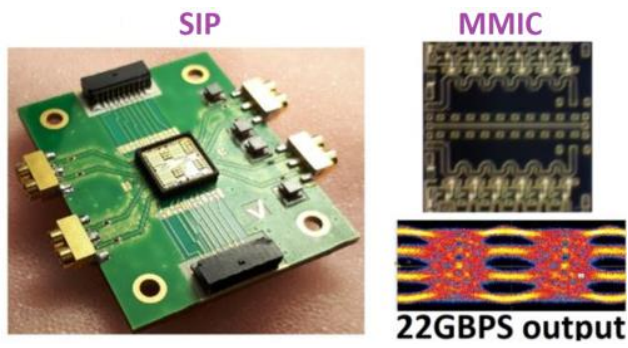

Fig. 6: Linear quad driver

wavelength changes last less than 500ns. We tested the middle and the extreme frequency of the C-band as visible on the laser output optical spectra (Fig. 7): $\lambda 0$ (193.1THz), $\lambda 1$ (193.53THz),

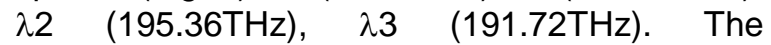
Defragmentation VNF is able to calculate the best sequence of lightpaths migration, in order to minimize the number of channels to be switched off and on again. After the gain estimation of the spectrum occupancy after and before migration, all the defragmentation process is running automatically, and flexi-grid allocation table is

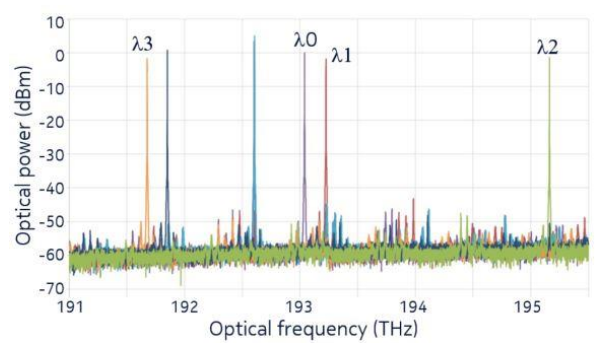

Fig. 7: Optical spectrum from high speed tunable laser displayed as a result (Fig. 5) with the new spectral slots occupancy for the same lightpaths list.

\section{Conclusions}

We proposed an end-to-end solution to perform and automate optical defragmentation. As a VNF in a fully modular SDN controller environment, it is linked to a hardware prototype of flexible transmitter with high-speed tunable transmitter and elastic programmable interface. Wavelength switching on all C-band is measured below $500 \mathrm{~ns}$ at the laser driver board level and below $0.5 \mathrm{~ms}$ from the application level.

\section{Acknowledgements}

The authors would like to thank the Celtic-Plus SENDATE-TANDEM project and the partners for the fruitful discussions.

\section{References}

[1] M. Jinno, H. Takara, Y. Sone, K. Yonenaga, A. Hirano, "Multiflow optical transponder for efficient multilayer optical networking," in IEEE Commun. Mag., vol. 50, no.5, May 2012.

[2] O. Gerstel, M. Jinno, A. lord, S.B. Yoo, "Elastic optical networking: A new dawn for the optical layer?," in IEEE Commun. Mag., vol. 50, no.2, 2012.

[3] P. Layec, A. Morea, F. Vacondio, O. Rival, and J.-C. Antona "Elastic Optical Networks: The Global Evolution to Software Configurable Optical Networks," in Bell Labs Technical Journal, vol. 18, no.3, pp. 133-151, Dec. 2013.

[4] Z. Zhu et al. "OpenFlow-Assisted Online Defragmentation in Single-/Multi-Domain Software-Defined Elastic Optical Networks," in JOCN, vol. 7, no.1, 2015

[5] ONOS consortium, http://onosproject.org

[6] Q. Van Phan et al., "Virtualized Routing and Frequency Allocation Functions in Elastic Optical Networks", W1.F.6, ECOC2017.

[7] A. Dupas, et al., "Real-Time Demonstration of SoftwareDefined Elastic Interface for Flexgrid Networks", in Proc.OFC 2015, paper M3A.2.

[8] A. Dupas et al., Ultra-fast Hitless 100Gbit/s Real-Time Bandwidth Variable Transmitter with SDN optical control" in Proc. OFC 2018, Th2A.46, San Diego.

[9] http://www.vectrawave.com/ 